\title{
XIX.
}

Aus der chirurgisehen Abteilung des städtischen Krankenhauses im Friedrichshain zu Berlin (Direktor Dr. A. Neumann).

\section{Ein Weg zum vesicalen Ureterende beim Manne.}

\author{
Yon \\ Dr. Alfred Neumann. \\ (Mit Tafel II.) \\ (Nach einem Vortrage in der "Freien Vereinigung der Chirargen Berlins" \\ am 13. Juni 1904.)
}

Zur Freilegung des Ureters sind die mannigfaltigsten Wege eingeschlagen worden. Diese Wege sind verschieden, je nach dem $\mathrm{Ab}$ schnitt des Ureters, den man in Angriff nchmen will, sei es, dals es sich darum handelt, Steine aus dem Lumen desselben zu entfernen oder krankhaft veränderte Partien des Creters selbst oder in der Nähe desselben zur Heilung zu bringen.

Man teilt in dieser Hinsicht praktisch den Ureter im wesentlichen in vier Abschnitte, und zwar erstens in den dem Nierenbecken direkt benachbarten Teil, in den dann folgenden Abschnitt bis zur Kreuzung mit den Vasa iliaca, also bis zum Eintritt in das kleine Becken, ferner in den längs der Seitenwand des kleinen Beckens verlaufenden Teil und schließlich in den der Blase direkt benachbarten Abschnitt desselben.

Für die außerhalb des kleinen Beckens an der hinteren Bauchwand gelegenen $\Lambda$ bschnitte des Ureters ist in den meisten Fällen der zweckmäligste und der am meisten betretene Weg der Schrägschnitt, wie er durch von Bergmann angegeben worden ist, ja, in einer großen Reihe von Fällen, namentlich wenn dieser Schnitt, wie das Israel ausgeführt hat, weiter nach vorn geführt wird, bis zur Mitte des Poupartischen Bandes und noch weiter bis zur Medianlinie, gelingt es mit der von Bergmannschen Schnittführung, den gesamten Creter frei zu legen, denselben extraperitoneal zn exstirpieren, 
oder anch, wie bekanntlich ebenfalls Israel gelehrt hat, extraperitoneal eine Lretero-cysto-an stomose herzustellen.

Die von Bergmannsche Schnittührung jedoch zur extraperitonealen Freilegung des Lreters setzt voraus, daß es möglich ist, von dem Hautschnitt aus das Peritoneum und mit ihm den Ureterstumpf yon der hinteren Bauchwand loszulösen. Ist diese Trennbarkeit des Peritoneum von der hinteren Baucbwand verloren gegangen, sei es durch entzïndliche, sei es durch neoplastische Veränderungen des das Peritoneum an seiner Unterlage anhaftenden Bindegewebes, dann ist die Freilegung des Creters ohne Eröffnung der Peritonealhöhle meist nicht möglich. Diese mehr oder minder unbeabsichtigte Eröffnung der Peritonealhöhle hat nicht viel auf sich, wenn sie auberhalb des keinen Beckens stattfindet, dort wo das entstandene Loch dem Auge zugänglich gemacht werden und lege artis versorgt werden kann, und wo eine litverletzung anderer wichtiger Organe, namentlich größerer Blutgefälie, nicht zu befürchten ist. Schwieriger liegen die Verhältnisse im kleinen Becken. IIandelt es sich darum, hicr am Lreter inmitten von entzündlichen oder neoplastischen diffusen Verwachsungen zu operieren, wie z. B. bei Ureterscheidenfisteln, bei Umwachsungen des Ureters durch Tumorgewebe, dann wird man andere Wege einschlagen müssen.

Es kann nun nicht meine Absicht sein, diese anderen Wege einzeln zu besprechen. Es sei mir nur gestattet, einen nicht gewöhnlichen Weg zu schildern, anf welchem ich mir in einem verzweifelt liegenden Falle bei einem Manne den Zugang zu der seitlichen hinteren Blasenwand verschaffte.

Die Krankengeschichte, welche auch sonst manches Interessante bietet, auf welches ich näher bier nicht eingehen will, ist kurz folgende:

Der Tranke. ein 26 jähriger Landwirt, hatte mit 13 Jahren das Unglïek, rom Pferde za stürzen. In direktem Ansehlusse an dieson Unfall rachte er ein kurzes Krankenlager durch. Fr hatte heftige Schmerzen in der linken Seite, laäufiges Erbrechen und hartnäekige Stuhlverstopfung. Dieses Krankheitsliild mit paroxysmal auftretenden Sehmerzen in der linken Lumbalgegend und mit Ileuserscheinungen wiederholte sich in typiseher Weise 2 Jahre, 3 Jahre and 4 Jahre nach dem Unfalle und zwar immer nach einem Abusus spirituosorum. Der letzte Anfall in Jahre 1895 führte itn in unser Krankenhaus. Wieder nach dem Genuß kalten Weines waren die alten Selmerzen in der linken Lumbalgegend aufgetreten. Dieses Mal aher waren die begleitenden ileusartigen Symptome, das Erbrechen so heftig, die Verstopfung für Stuhl und Winde so anhaltend und quälend, daß Patient schwer kollabiert war. $\mathrm{Als}$ dazu noch bei uns bei der Cntersuchung per rectum ein kinderarmdicker, wurstförmiger Tumor, welcher an der linken Beckenwand lateralwärts und nach oben aus dem Becken herausging, ga- 
fïllt wurde, wurde das Vorhandensein einer Darminvagination angenommen und die Laparotomie durch Herrn Geh. Rat If ahn vorgenommen. Diese ergab einen überrasehenden Befund. Ein mechanischer Ileus lag nicht vor. Die Ileuserseheinungen waren nur reflektoriseh ansgelöst worden. Der per rectum nach oben und auß3en ziehende wurstförmige 'Tumor war nichts anderes als der zu Kinderarmstärke ausgedehnte linke Creter, der nach der bläulichen Farbe, mit welcher er durch das bedeckende Peritoneum hindurchschimmerte, zn schließen, von der Blase bis zum Xierenbecken mit Blat gefüllt war. An der linken Niere selbst wurde damals nichts Pathologisches konstatiert.

Die Bauchhölle wurde wieder geseschlossen. Am nächsten Tage entleerte der Kranke eine große Menge von Blutgerinnseln durch die Harnröhre. 2 Tage nachher gingen Winde und bald daranf auch Stuhlgang ab. Der Crin euthielt noeh 6 'Tage lang rote Blutkörperehen und etwas Albumen. Dann war auch der Lrin normal. 5 Wochen nach der Laparotomie wurde der Patient entlassen; am Tage der Entlassung fiilhite man an der Stelle des Tumors einen derben Strang, der absolut druekunempfincilich war.

Es ist anzunehmen, daß bei dem Lnfalle irgendwie der linke Ureter dicht an der Blasenmündung verletzt worden war, daß an der Verletzungsstelle ein Passagehindernis, eine Striktur oder Knickung und daß ferner eine Neigung zu Urinverhaltung im Ureter mit Blutungen in denselben und zwar wahrseheinlich aus der Niere selbst zurïekgeblieben waren.

Volle 7 Jahre nachher fühlte sich der Kranke völlig sesund und frei von Beschwerden. Im Februar 1902 acquirierte er eine Gonorrhöe, bekan dazu heftige Blasenbeschwerden und suchte im März 1902 unser Krankenhaus wieder auf.

Der gonokokkenhaltige eitrige AusfluB aus der Ifarnröhre ließ bald nach, auch die akuten Erscheinungen des Blasenkatarrhs, Schmerzen beim Lrinlassen, Blasentenesmen traten zurïck, der worher alkalische Lrin warde sauer. Doch blieb derselbe stark eiterhaltig, und es traten immer mehr die Symptome einer eitrig fibrinösen Ureteritis in den Vordergrund. Alle paar Tage gingen unter heftigen kolikartigen Schmerzen federkiel- bis kleinfingerdicke und ebenso lange Gerinnsel mit dem Urin ab.

Per rectum fühlte man wieder links neben der Blase an der Stelle des linken Ureters einen wurstförmigen Strang, welcher unter den Fingern hin und her glitt und besonders an einer Stelle neben einer erbsengrolsen Lymphdrüse sehr druckempfindlich war.

Das Krankheitsbild besserte sich nicht, es traten heftige Schmerzen in der Gegend der linken Niere, die als vergrößsert zu fühlen war, anf, Fieber trat hinzu, und der Kranke kam sehr herunter.

Am 28. Mai 1902 wurde ron Herrn Geheimrat $\mathrm{Hahn}$ durch Schrägschuitt die linke Niere freigelegt, herausgelagert und durch Sektionssehnitt gespalten.

Die Niere war vergrößert, die Nierensubstanz derb, stark reduziert und von kleinen Cysten durchsetzt. In den Cysten fand sieh, ebenso wie in dem erweiterten Nierenbecken hämorrhagischer Fiter. Beim Versuch, den Ureter zu sondieren, konnte die Sonde nur $10 \mathrm{~cm}$ weit eingeführt werden. 
Da die Funktionsfähigkeit der rechten Niere wegen des starken Fitergehaltes des Crins vorher nicht festzustellen war, wurde die Niere tamponiert und zuriickgelagert, und nachdem die rechte Niere durch den Harnleiterkatheterismus und die Phloridzinreaktion als intakt befunden war, warde (durch den Is ra el schen verlängerten Schrägschnitt) an 2 I. Juni 190? die linke Niere und der linke Treter bis etwa $4 \mathrm{~cm}$ ron der Blase entfernt exstirpiert.

Die Ireterwand war etwa $2 \mathrm{~mm}$ dick, das Lumen lief sich bis etwál. Handbreite von der Blase bougieren, dann stieß das Bougie anf ein unüberwindliches Hindernis, hinter welchem der Creter eine otwa wallnußgroße ampullare Erweiterung aufwies.

Der an der Blase zurückgebliebene Lreterstumpf war unterbnnden worden, die ausgedehnte Wundhöhle wurde sorgfältig; drainiert und tamponiert.

Trotzdem war es nicht zu vermeiden gewesen, dals sich in Ansehlub an diese Operation, wahrscheinlich ron dem Ureterstumpf ans, das Krankheitsbild einer schweren jauchigen Phlegmone des lockeren, zwischen Blase und Mastdarm befindlichen Gewebes entwickelte mit Fiebersteigerungen bis 40 und 41 Grad, Pulsfrequenzen bis 120 und 132 , Benommenheit, Delirien und leichten peritonitischen Keizerscheinungen, Eischeinungen, die auch nach woiterer Albösung des Peritoneum ron dem subperitonealen Gewebe nicht nachlieben und erst besser wurden, nachdem der phlogmonösen Eiterung durch Eröffnung von der vorderen Rectumwand aus Abfluß geschaffen und ein Drain von der Bauchwande in das Becken hineingeführt worden war.

Nun folgte ein 7 monatliches Krankenlager, während welchom vergebens versucht wurde, der profusen Eiterung um den Creterstumpf Herr zu werden. Die akuten Entzündungserscheinungen gingen vollkommen zurüek, die große linksseitige Flankenwunde heilte bis auf den Kanal für das Drain vollkommen zn. Jedoch weder Spülungen mit allen möglichen Flïssigkeiten, anch nicht die permanente Irigation durch das Drain, noch die Spaltang des Sphineter ani und die breite Frweiterung und Tamponade der Öffnung an der vorderen Rectumwand führten zu einer wesentlichen Ferminclerung der Fiterung. Lrin floß nie durch das Drain ab. Doch sobald das Drain sich verstopfte oder verschob, oder aus Furcht vor der Esur der von demselben gelkrenzten freiliegenden Iliacalgefäße vollkommen fortyelassen wurde, drangen fäculenter Fiter nnd fäculent riechende Gase in die Blase und durch die Harnoohre nach außen. Patient kam außerordentlich herunter, und es mußte unter allen Umständen Abhilfe geschaffen werden.

Die insufficient gewordene Ligatur des Ureterstumpfes neu anzulegen, war gewiß erwünseht, doch da kein Lrin aus der Blase in die Absceßhöhle abfloß, nicht nötig. Es mußte genügen, den Absceß breit zu eröffnen, ohne ihn mit dem Mastdarmlumen in Kommunication zu bringen, die $A$ bsceßwand auszukratzen und die Höhle geniggend zu tamponieren.

Wo lag nan genau der Abseeß? Wenn wir das Bild des Becken- 
querschnittes von Jössel und Waldeyer betrachten (Abbildung 1), so können wir uns über die genauere Lage des Abscesses orientieren.

Der AbsceB mußte gelegen sein an der Stelle der hinteren Blasenwand um diejenige Strecke des Ureters herum, zwischen der Uretermündnng in die Blase und derjenigen der Stelle, an welcher der Ureter auf der Abbildung 1 durchschnitten dargestellt ist.

Der wohl am mcisten betretene $\mathrm{Weg}$, um an diese Stelle des Ureters zu gelangen, ist der subperitoneale von einem lumbo-iliacalen Schrägschnitt her. Doch dieser Weg war in unserem Falle nicht mehr gangbar, weil das l'eritoneum durch die monatelange Eiterung mit der Unterlage fest verwachsen war.

Eine einfache Erweiterung des Fistelganges daselbst aber, die versucht wurde, mußte wegen der callösen Wandungen desselben und der Gefahr der Eröffnung der Peritonealhöhle wieder aufgegeben werden.

Der Zugang vom Innern der Blase selbst her nach $\Lambda$ usführung der Sectio alta wäre möglich; doch man würde dann an Stelle einer gut schließenden Lreterblasenklappe eine Pforte in den Absceß schaffen, durch die der Urin frei in die Jauchehöhle eintreten könnte. Man würde aus dem paravesicalen Absceß ein jauchendes Blasendivertikel machen und damit eine jauchende Cystitis, die um so gefährlicher war, als der Patient nur noch einen Creter und eine Niere hatte.

Von der Peritonealhöhle aus per laparotomiam den Absceß zu öffnen, war bei der ungünstigen Lage tief im Douglas ohne Gefahr einer tödlichen Peritonitis nicht angängig.

Auch der parasacrale Weg vom Foramen ischiadicum aus oder der sacrale nach Resection des Kreuzbeines schien zu gefährlich, einmal weil der Absceß von der Kreuzbeinoberfläche zu entfernt lag; vorn an der Vorderwand des Mastdarmes, und zweitens, weil der schwer vermeidbare Kontakt des inficierten Eiters mit der vielbuchtigen Wundhöhle eine septische allgemeine Infektion allzusehr befürchten ließs.

So blieb nur noch der perineale Weg übrig.

Bekanntlieh waren es vor allen Dittel und Zuckerkandel, die diesen Weg methodisch ausgebaut haben. Auf demselben gelingt es, nicht nur die gesamte Prostata, sondern auch den unteren Teil der Samenblasen und den in dem Winkel zwischen den beiden Vasa deferentia liegenden Teil des Blasengrundes zugänglich zu machen. Doch war es mir in unserem Falle von vornherein sehr zweifelhaft, ob ich auf diesem Wege ohne Gefahr bis in den Absceb würde vordringen können. 
Ich beschloß diesen Weg zu versuchen, und sollte er nicht zum Ziele führen, auf einem Wege weiter zu gehen, den ich in der Lnfallpraxis des öfteren kennen gelernt leatte and der auch durch die anatomischen Verhältnisse vorgezeichnet war.

Wie ich ${ }^{1}$ ) bereits vor Jahren bei Gelegenheit der Besprechung. der in unserem Krankenhause beobachteten Pfählungsverletzungen hervorgehoben habe, gehen die von der unteren Beckenapertur vordringenden Körper mit Vorliebe ohne die Bauchhöhle zu eröffnen, vom Damme her an der seitlichen Beckenwand entlang in die Muskulatur derselben. Dieses Verhalten der von unten andringenden pfählenden Körper wird in erster Limie bedingt durch die nach unten zu convergierenden Wände des knöchernen Beckenringes, wie ich damals ausgeführt habe, in zweiter Linie scheinen mir aber die pfählenden Körper, wenn sie den muskulösen Beckenboden des Trigonum urogenitale durchbohrt haben, deswegen so häufig in die Muskulatur der seitlichen Beckenwand abzawcichen, weil sie durch den eigenartigen Verlauf der consistenteren Fascien, nämlich der Fascien des M. obturator int. und des M. levator ani, gewissermaßen dahin geleitet werden.

Wenn wir auf das ebenfalls aus dem Atlas von Jössel und Waldeyer entnommene Bild (Abbildung 2) eines Frontalsehnittes durch das Becken einen Blick werfen, so sehen wir folgendes:

Das Bild ist nach einem Gefrierschnitt gezeichnet. Der Schnitt geht frontal durch die Harnblase und den prostatischen Teil der Harnröbre. Am knöchernen Beckenring ist der Pfannengrund des Hüftgelenkes und das Foramen obturatorium getroffen. Dem knöchernen Beckenringe anliegend und das Foramen obturatorium von innen deckend, sieht man den M. obturator int., dessen oberer Ursprung weiter nach vorn, als es das Bild zeigt, an der Innenfläche des horizontalen schambeinastes liegt. Nach innen von der unteren Irälfte des M. obturator int. liegt der M. levator ani, dessen dargestellter Quersehnitt als stumpfwinkliges Dreieck gezeichnet ist, mit einem oberen spitzen Winkel, welcher der oberen Ansatzstelle des Muskels, seinem quer über die Innenfläche des M. obturator int. verlaufenden Arcus tendineus entspricht, einem äuferen unteren stumpfen Winkel und einem inneren unteren spitzen Winkel, welch letzterer der Spitze der Prostata benachbart ist und dem Rande des Schlitzes entspricht, welchen der Levatortrichter vorn für den I)urchschnitt der Harnröhre frei läßt.

Die kurze, zwischen den beiden unteren Winkeln verlaufende Seite des Dreieckes ruht der Oberfläche des Trigonum urogenitale auf.

1) A. Neumann, ẗber die im Krankenhause im Friedrichshain von 1880 jis 1.595 beobachteten Pfählungen. Deutsche med. Wochenschr. 1899. Nr. 33.

Deatsche Zeitschrift f. Chirurgio. LXXIV. Bd. 
Uns interessiert hier vor allem das Verhalten der parietalen Beckenfascie. Wenn ich mich an die Darstellung ron Jössel und Waldeyer halte, so ist die parietale Beckenfascie als die Fortsetzung der großen Fascia endoabdominalis anzusehen. Diese Fascie geht, wenn sie in das kleine Becken eintritt, über auf die Innenfläche des M. obturator int., mit der Eigenfascie dieses Muskels verschmelzend, teilt sich dann an der Stelle, an welcher der M. levator ani an der Innenseite des Obturator int. mit seinen Sehnenbogen quer entspringt. gewissermaljen in zwei Fascienblätter, von denen das eine als eigentliche Fortsetzung der parietalen Beckenfascie die Innenfiäche des Levatortrichters bekleidet, das andere Blatt zwischen M. levator ani und M. obturator int. verlaufend, die sich berührenden Eigenfascien der beiden benachbarten Muskeln darstellt. „An der medialen unteren Ecke des M. levator ani ist ein wichtiger Punkt für die Beckenfascie. Sie trifft hier zusammen mit der unteren Fascie des M. levator ani, welche vorn dicht der Fascia obturatoria aufliegt; ferner mit der letzteren selbst, welche, auf der oberen Fläche des M. trigoni urogenitalis entlang, beim Manne bis zur Harnröhre und Prostata - sich hinzieht und dort, ebenso wie die parictale Beckenfascie, in die

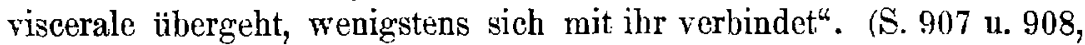
Jössel und Waldeyer).

Ich faßte den Plan, für den Fali, daß es nicht gelingen sollte, einfach rom oberen Teil der Prostata her den Absceß zu eröffnen, olgendermaben vorzugehen: In der Höhe der unteren Spitze der Prostata nach links vorn abzuweichen entlang dem Trigonum urogenitale und der dasselbe von oben bedeckenden Fascie und stumpf unter die Fascie des M. obturator int. einzudringen, zwischen dieser Fascie und dem M. obturator int. möglichst hoch nach oben vorzudringen und dann die so geschaffene Höhle von oben her durch einen flachen Hautschmitt längs des linken horizontalen Schambeinastes zu eröffnen. Es mußte so gelingen, die Stelle des Abscesses, der ich s u b perito neal wegen der vorausgegangenen Fiterung nicht beikommen konnte, gewissermalien subfascial freizulegen.

Stärkere Blutungen hatte ich nicht zu befürchten, wenn ich mich nach innen von den Iliacalgefäßen hielt, da, wie $\mathrm{J} \ddot{\text { sssel }} \mathrm{l}$ und $\mathrm{Wal-}$ deyer besonders hervorheben, die von den hypogastrischen Gefälen ausgehenden größeren Seitenäste alle nach innen von der Fascie liegen.

Nachdem ich mich durch Leichenversuche von der Gangbarkeit des Weges überzeugt hatte, schritt ich am 14. Februar 1903 zur Operation.

Als Hautschnitt wählte ich den Kocherschen queren Spitzbogenschnitt. Es gelang von diesem Schnitt aus leicht ohne erheblichen Blut- 
veriust bis zum oberen Rande der Prostata vorzudringen. Dann aber stieß ich auf so derbes schwieliges Gewehe, daß ein Cnterscheiden der einzelnen Gewebeschichten unmöglich war, und ich befürchten mußte, vor diem Abszeß die Blase oder die Peritoneallöhle zu eröffnen.

An der Vorderfläche der Wundhöhle markierten sieh dentlieh die quer verlaufenden Faseri des $M$. transversus perinei profundus als lintere Begrenzung des Trigonum mogenitale. Zwischen diesen und der Spitze der Prostata links an derselben vorbei war es ein leichtes, mit dem Finger stumpf gegen die chalakteristische Muskelmasse des M. obturatur int. vorzudringen und von der Oberfläche dieses Muskels in der geschilderten Weise die Fascie abzulösen in einer Ausdehnung, daB ich bequem zwei Finger meiner rechten Hand hin und her und bis zum linken horizontalen Schambeinast nach oben schieben konnte. Der Kranke wurde dann aus de: Steinschnittlage in die horizontale Rückenlage gebracht, der in den Abszeß fiihrende Fistelgang sorgfältig mit Jodoformgaze austamponiert. Dann durchtrennte ich durch einen in der Richtung des Poupartischen Bandes verlaufenden Schnitt, welcher etwa 2 Querfinger breit nach unten ron der Fistelöffnung, etwa an der Spina iliaca ant. sup. begann und bis zur Symphyse reichte, Haut und Muskulatur, vertiefte dann diesen Schnitt, mich medianwaits von den Iliacalgefäßen haltend, bis auf das Periost des Inrizontalen Schambeinastes. Einen kleinen Einril des Peritoneums, den ich nicht vermeiden konnte, nähte ich sofort wieder zu und kam dann mit Leichtigkeit in die vorher geschaffene durch Gazetampons markierte Höhle. Nach stumpfer Erweiterung des oberen Zuganges der Höhle nach der Symphyse gelang es leicht, dic von der Dammwunde eingeführten Finger mit denen von del Regio pubica eingeführten miteinander in Berührung zu bringen, an đer lateralen Partie der Hölle die Beckenweichteile und in derselben den ganzen Verlauf des tamponierten Fistelganges und am unteren Ende desselben ganz in der Tiefe den als derbe Resistenz markierten, bis jetzt uneröfneton Abszeß in der Gegend des Lreterstumpfes abzutasten. Ich tamponierte die ganze große Höhle aus, wartete dann 14 Tage, bis sich die Wand derselben mit guten Granulationen ausgekleidet hatte und auch der genähte Peritonealrif genügend sicher verklebt war. Dann ging ich mit emer langen Kornzange durch den Fistelgang in den AbszeB ein, stieh lie Spitze der Kornzange gegen den in die subfasciale Wandhöhle eingefiihten Zeigefinger der linken Mand vor und erweiterte die so entstandene Offnung durch ein Bruchmesser. Jetzt konnte ich, nachder sicl reichlich stinkender Fiter entlecrt hatte, bequen die ganze hühnereigroße Abszeßhöhle abtasten, sorgfältig curettieren, tamponieren und drainieren.

Der weitere Krankeitsverlauf war ein vollkommen glatter. Die Sekretion aus der Abszeßhöhle war ron nun an gering. Der Crin war zwei Tage nach der Operation blutig, vom dritten Tage an vollkommen kiar. 14 Tage nachher konnte das Drain fortgelassen werden, die AbszeB-Rectalfistel hatte sich vorher geschlossen. Am 6. Mai 1903, 2 Monate nach der Operation, lionnte der Kranke, vollkommen beschwerdefrei, mit klarem, wenig Hiterkörperchen enthaltenden Crin, außerordentlich erholt, entlassen werden.

Er war später wegen einer leichten Trübung des Crins noch einmal einige Tage in unserer Beobachtung. Im übrigen ist ex seit einem Jahre volkommen gesund und ohne Beschwerden als Landwirt tätig gewesen. 